\title{
Étude phytochimique et évaluation du potentiel antioxydant de deux cultivars de Piper nigrum L. cultivés en Côte d'Ivoire
}

\author{
DJIWONOU Koffi Jean-Baptiste ${ }^{1}$, CHERIF Aïcha², N'GAMAN-KOUASSI Christelle², DOGBO Dénézon \\ Odette', MAMYRBEKOVA-BEKRO Janat Akhanovna*2, BEKRO Yves-Alain² \\ 1 Laboratoire de Biologie et Amélioration des Productions Végétales (LBAPV), UFR-SN \\ ${ }^{2}$ Laboratoire de Chimie Bio-Organique et de Substances Naturelles (LCBOSN), URF-SFA \\ Université Nangui Abrogoua 02 BP 801 Abidjan 02 (Côte d'Ivoire) \\ *Auteur correspondant : kojanova1926@hotmail.fr, kohuechristelle@yahoo.fr
}

Original submitted in on $9^{\text {th }}$ September 2019. Published online at www.m.elewa.org/journals/ on $31^{\text {st }}$ December 2019 https://doi.org/10.35759/JABs.v144.3

\section{RÉSUMÉ}

Objectif: Piper nigrum L. ou poivre noir est connu pour ses vertus médicinales. Deux de ses cultivars, cultivés en Côte d'Ivoire ont retenu notre attention dans le présent travail. Le principal objectif a été de déterminer la composition phytochimique desdits cultivars et d'apprécier leur potentiel antioxydant à l'égard du radical DPPH.

Méthodologie et résultats : Les tests qualitatifs et quantitatifs ont montré la coprésence de phytocomposés bio-importants (coumarines, flavonoïdes, anthocyanes, alcaloïdes, stérols, triterpènes de type lupane) dans les cultivars MK et LG. De plus, la quantification des constituants phénoliques montre des teneurs notables en phénols totaux de 89,082 et $74,492 \mu \mathrm{gEAG} / \mathrm{g}$ et en flavonoïdes totaux de 49,456 et $55,343 \mu \mathrm{gEC} / \mathrm{g}$ d'extrait respectivement pour MK et LG. Leur potentiel anti radicalaire par rapport au DPPH a été déterminé et les valeurs de la $C E_{50}$ de LG et de MK sont respectivement de 1,209 et 1,432.

Conclusion et application des résultats : Cette étude montre d'une part, que les cultivars de Piper nigrum renferment des phytosubstances importantes, et d'autre part, met en évidence leur potentiel antioxydant manifeste en comparaison à celle connue de la quercétine, prise comme référence. Cette vertu constatée trace une suite d'étude qui fera de cette matrice végétale une source d'antioxydants naturels. Nous espérons par la présente étude avoir contribué à initier un début vers la conception d'un complément alicamentaire à vertu antioxydante.

Mots-clés : Piper nigrum, cultivars, composition phytochimique, DPPH. 
Phytochemical study and antioxidant potential of two cultivars of Piper nigrum L. cultivated in Côte d'Ivoire

\section{ABSTRACT}

Objective: Piper nigrum L. or black pepper is known for its medicinal properties. Two of his cultivars, cultivated in Côte d'Ivoire, caught our attention in this work. The main objective was to determine the phytochemical composition of these cultivars and to assess their antioxidant potential with regard to DPPH.

Methodology and results: The results of qualitative and quantitative tests showed the co-presence of biologically important phytocompounds (coumarins, flavonoids, anthocyanins, alkaloids, sterols, lupane triterpenes) in MK and LG cultivars. In addition, quantification of phenolic constituents showed significant total phenol contents of 89.082 and $74.492 \mu \mathrm{gEGA} / \mathrm{g}$ and total flavonoids of 49.456 and $55.343 \mu \mathrm{gEC} / \mathrm{g}$ extract respectively for MK and LG. Their DPPH potential scavenging activity has been determined and the $E_{50}$ values for $L G$ and $M K$ are 1.209 and 1.432, respectively.

Conclusion and application of results: This study shows, on the one hand, that Piper nigrum cultivars contain important phytosubstances, and on the other hand, demonstrates their obvious antioxidant potential in comparison with that known from quercetin, taken as a reference. This observed virtue traces a series of studies that will make this plant matrix a source of natural antioxidants. We hope by this study to have helped to initiate a start towards the design of a food supplement with antioxidant virtue.

Keywords : Piper nigrum, cultivars, phytochemical composition, DPPH.

\section{INTRODUCTION}

Le poivre est le fruit du poivrier (Piper nigrum L), connu sous le nom de poivre noir ou «black Pepper ». Ce fruit est considéré comme la « reine des épices " en raison d'une part, de son usage dans l'industrie des épices, et d'autre part, de sa valeur marchande sur le marché international au regard de sa teneur en terpénoïdes ( $\alpha$-pinène, $\beta$ pinène, $\delta$-carène, limonène, a-terpinène-4-ol, cymène) et en alcaloïdes (pipérine et composés apparentés) antioxydants et antimicrobiens (Srinivasan, 2007). Originaire des régions tropicales et subtropicales de l'Inde plus précisément de la côte Ouest de Malabar de l'état du Kerala (Nair et al., 2003; Khan et al., 2010 ; Hussain et al., 2011), le poivre noir s'est retrouvé dans les autres régions tropicales au cours du commerce triangulaire, et par la suite a gagné les côtes ivoiriennes pendant la colonisation (Borget, 1991). Compte tenu de son importance se traduisant par son usage dans les systèmes médicaux asiatiques (Scott et al., 2008) et comme

\section{MATÉRIEL ET MÉTHODES}

Matériel végétal; ॥ est constitué des graines de cultivars Lompung (LG) et Montuk (MK) de Piper nigrum L., récoltées dans une exploitation agricole d'Azaguié ( $5^{\circ} 38^{\prime} 00^{\prime \prime}$ nord, $4^{\circ} 05^{\prime} 00^{\prime \prime}$ ouest), une ville antioxydant (Nahak and Sahu, 2011), antimutagène et anti carcinogène (Ahmad et al., 2011), antimicrobien, diurétiques et antiseptique (Achinewu et al., 1995), cette épice a fait l'objet de nombreux travaux scientifiques. Dans de nombreux cas, l'effet bénéfique des plantes sur la santé est lié aux alcaloïdes, aux terpènes et aux polyphénols qu'elles renferment. Au nombre de ces métabolites secondaires, les polyphénols largement distribués dans le règne végétal, sont reconnus depuis longtemps comme de puissants antioxydants par excellence. La présente investigation a été conduite dans le but de déterminer la composition phytochimique des extraits issus de deux cultivars de Piper nigrum L., de quantifier leurs teneurs en polyphénols, flavonoïdes, anthocyanes, aglycones flavoniques, tanins condensés et hydrolysables et enfin d'évaluer leur potentiel antioxydant à l'égard du DPPH.

de la Région de I'Agnéby-Tiassa, située au Sud de la Côte d'Ivoire dans le département d'Agboville ( $5^{\circ} 55^{\prime} 41^{\prime \prime}$ nord, $4^{\circ} 13^{\prime} 01^{\prime \prime}$ ouest). Les cultivars ont été identifiés au Centre National de Floristique d'Abidjan. 
Les graines ont été nettoyées à l'eau courante, séchées à la température ambiante à l'abri de la lumière pendant 15 jours, puis conservées dans une étuve à $45^{\circ} \mathrm{C}$ durant 3 jours. Les graines séchées ont été réduites en poudre avec un broyeur électrique, et conservées dans des bocaux en verre hermétiquement fermés.

Préparation des extraits : $15 \mathrm{~g}$ de poudre obtenue à partir des graines broyées de chaque cultivar ont macérés dans $30 \mathrm{ml}$ de méthanol $(80 \%)$ pendant $24 \mathrm{~h}$ à température ambiante. Après filtration sous vide, les filtrats obtenus après 3 épuisements successifs au solvant d'extraction, ont été conservés au réfrigérateur $\left(4^{\circ} \mathrm{C}\right)$ pendant $24 \mathrm{~h}$. Après décantation et concentration à l'aide d'un évaporateur rotatif $(\mathrm{BÜCHI})$, les extraits secs $L G$ et $M K$ ont été obtenus après conservation dans une étuve $\left(40^{\circ} \mathrm{C}\right)$ pendant $24 \mathrm{~h}$.

Analyse qualitative: tests d'identification par chromatographie sur couche mince (CCM)

Les tests d'identification ont été réalisés sur les extraits sélectifs de LG et MK obtenus par extraction solideliquide, suivant les méthodologies rapportées par (Ladiguina et al., 1983 ; Dohou et al., 2003 ; Békro et al., 2007 ; N'Gaman, 2013). Les extraits secs LG et MK ont été successivement épuisés avec l'hexane, le chloroforme $\left(\mathrm{CHCl}_{3}\right)$, l'acétate d'éthyle (AcOEt), le nbutanol $(\mathrm{n}-\mathrm{BuOH})$. Les révélateurs utilisés pour l'identification des groupes phytochimiques ont été le réactif Liebermann-Bürchard et la vanilline sulfurique à $5 \%(\mathrm{~m} / \mathrm{v})$ pour les terpènes et stérols, l'hydroxyde de potassium en solution méthanolique à $5 \%(\mathrm{~m} / \mathrm{v})$ et l'acétate de plomb basique pour les coumarines, l'ammoniac pour les coumarines, et flavonoïdes, le chlorure d'aluminium en solution aqueuse à $1 \%(\mathrm{~m} / \mathrm{v})$ pour les flavonoïdes, le réactif de Dragendorff pour les alcaloïdes. Les plaques chromatographiques (support aluminium, gel de silice $60 \mathrm{~F}_{254}$, épaisseur $0,2 \mathrm{~mm}$; Merck) ont été utilisées. Les empreintes moléculaires des métabolites secondaires ciblés ont été visualisées dans les chromatogrammes à l'œil nu et sous lumière UV à $365 \mathrm{~nm}$.

Analyse quantitative: La quantification des phénols totaux, des flavonoïdes et des tanins totaux, ainsi que des aglycones flavoniques et des anthocyanes a été réalisée conformément aux méthodes spectrophotométriques décrites dans la littérature (Lebreton \& Jay, 1967; Singleton et al., 1999; Heilerova et al., 2003 ; N'Guessan et al., 2011 ; Dif et al., 2015).

Evaluation du potentiel antioxydant: Le radical stable 2,2-diphényl-1-picrylhydrazyle (DPPH, Sigma Aldrich) a été solubilisé dans l'éthanol ( $\mathrm{EtOH})$ absolu pour obtenir une solution de concentration $0,03 \mathrm{mg} / \mathrm{ml}$. Différentes gammes de concentrations $(0,07 \mathrm{mg} / \mathrm{ml}$, $0,036 \mathrm{mg} / \mathrm{ml}, 0,0179 \mathrm{mg} / \mathrm{ml}, 0,00893 \mathrm{mg} / \mathrm{ml}, 0,004464$ $\mathrm{mg} / \mathrm{ml}$ et $0,0022321 \mathrm{mg} / \mathrm{ml}$ ) de chaque extrait ont été préparées dans le même solvant. Dans des tubes à essai secs et stériles, ont été introduits $0,4 \mathrm{ml}$ d'extrait, $1,2 \mathrm{ml}$ de DPPH et 2,4 ml d'EtOH. Après incubation des tubes à essai pendant $30 \mathrm{~min}$ à l'obscurité, les absorbances ont été lues à $517 \mathrm{~nm}$ avec un spectrophotomètre UV-visible (Jasco V500). La quercétine (Sigma Aldrich) a été l'antioxydant de référence employé (Espin et al., 2000 ; Slandjana et al., 2012). Le pourcentage de réduction du DPPH ( $R \%$ ) a été calculé suivant l'équation (1):

$R(\%)=\left(1-\left(\frac{\text { absorbance extrait }}{\text { absorbance control }}\right)\right) \times 100$

La demi-concentration efficace $\left(\mathrm{CE}_{50}\right)$ traduisant l'indice d'efficacité à différent temps des extraits est calculée selon l'équation (2) :

$C E_{50}\left(m g d^{\prime}\right.$ extrait $/ m g$ DPPH $)=\frac{C K_{50, t}}{\text { concentration initiale DPPII }}$

Où $\mathrm{CR}_{50, t}$ est la demi-concentration de réduction du DPPH déterminée graphiquement

\section{RESULTATS ET DISCUSSION}

Composition chimique des cultivars LG et MK : La CCM des extraits sélectifs de LG et de MK a permis de mettre en évidence leur composition chimique préliminaire respectivement (Tableau 1). Les stérols, terpènes et les triterpènes de type lupane ont été mis en évidence par le réactif de Liebermann-Bürchard dans les extraits hexaniques. Ce qui est en accord avec les nombreuses informations sur Piper nigrum L. tirées, se rapportant à sa richesse en huile essentielle (2-3\%) dont les constituants majoritaires sont des monoterpènes et sesquiterpènes ( $A 0$ et al., 1998; Chen et al., 2011 ; Xiang et al., 2017 ; Lui et al., 2018 ; Salehi et al., 2019). 
Tableau 1 : Phytocomposés identifiés dans les extraits hexaniques, chloroformiques, acétate d'éthyliques et nbutanoliques de Piper nigrum

\begin{tabular}{|c|c|c|c|}
\hline Extraits & $\begin{array}{l}\text { Phytocomposés } \\
\text { (révélateurs) }\end{array}$ & Cultivar Lompung (LG) & Cultivar Montuk (MK) \\
\hline \multirow{3}{*}{ 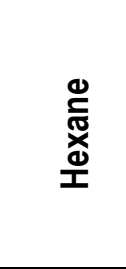 } & \multirow{2}{*}{$\begin{array}{c}\text { Stérols (L-B) } \\
\text { Triterpènes lupane (L-B) }\end{array}$} & $0,43(\mathrm{~B}-\mathrm{V}) ; 0,50(\mathrm{~V})$ & \multirow{2}{*}{$\begin{array}{c}0,58(\mathrm{~J}) ; 0,64(\mathrm{~J}) \\
0,40(\mathrm{Or}) ; 0,44(\mathrm{Or}) ; 0,54(\mathrm{Or}) ; \\
0,73(\mathrm{Or})\end{array}$} \\
\hline & & $0,58(\mathrm{~J}) ; 0,64(\mathrm{~J})$ & \\
\hline & Terpènes (L-B) & $\begin{array}{c}0,38(\mathrm{Vi}) ; 0,40(\mathrm{Or}) ; 0,50(\mathrm{~J}-\mathrm{Or}) \\
0,60(\mathrm{Or}) ; 0,76(\mathrm{~J}-\mathrm{Vi}) ; 0,89(\mathrm{Vi}) \\
0,91(\mathrm{Or})\end{array}$ & $\begin{array}{c}0,29(\mathrm{Or}) ; 0,39(\mathrm{Vi}) ; 0,51(\mathrm{~J}-\mathrm{Or}) \\
0,60(\mathrm{~J}-\mathrm{Or}) ; 0,71(\mathrm{~J}-\mathrm{Or}) ; 0,79(\mathrm{Vi})\end{array}$ \\
\hline \multirow{4}{*}{ 옹 } & \multirow{4}{*}{$\begin{array}{l}\text { Flavonoïdes }\left(\mathrm{AlCl}_{3}^{\mathrm{a}}, \mathrm{NH}_{3} \mathrm{~b}\right) \\
\text { Anthocyanes }\left(\mathrm{NH}_{3}^{\mathrm{b}}\right) \\
\text { Coumarines }\left(\mathrm{CH}_{3} \mathrm{COO}\right)_{2} \mathrm{~Pb} \\
\text { basique } \\
\text { Alcaloïdes (Dragendorff) }\end{array}$} & $\begin{array}{c}0,05(\mathrm{Ja}) ; 0,08\left(\mathrm{Ja}^{\mathrm{a}}\right) ; 0,23\left(\mathrm{~B}^{\mathrm{a}}\right) ; \\
0,31\left(\mathrm{~B}^{\mathrm{a}}\right) ; 0,42\left(\mathrm{~J}^{\mathrm{a}} \mathrm{B}\right) ; 0,48\left(\mathrm{~B}^{\mathrm{a}}\right) ; \\
0,56\left(\mathrm{JJ}^{\mathrm{b}}\right) ; 0,64\left(\mathrm{~J}-\mathrm{Or}^{\mathrm{a}}, \mathrm{Jb}^{\mathrm{b}}\right) ; 0,74(\mathrm{~J}- \\
\left.\mathrm{Or}^{\mathrm{b}}\right) ; 0,80\left(\mathrm{~J}-\mathrm{r}^{\mathrm{b}}\right) ; 0,89\left(\mathrm{Jb}^{\mathrm{b}}\right)\end{array}$ & \multirow{4}{*}{ 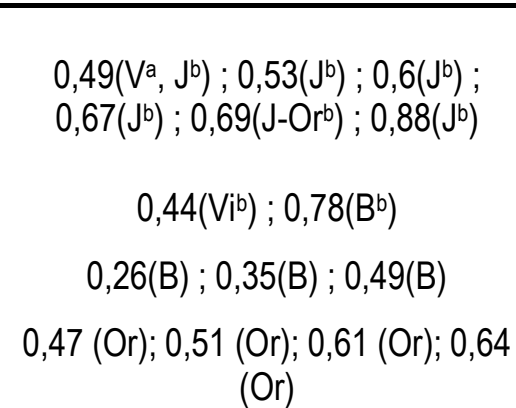 } \\
\hline & & $0,44\left(\mathrm{Vi}^{\mathrm{b}}\right)$ & \\
\hline & & $0,25(\mathrm{~B}) ; 0,42(\mathrm{~B}) ; 0,48(\mathrm{~B})$ & \\
\hline & & $\begin{array}{c}0,47 \text { (Or) ; 0,51 (Or) ; 0,61 (Or) ; } \\
0,64(\mathrm{Or})\end{array}$ & \\
\hline \multirow[t]{3}{*}{ 岀 } & $\begin{array}{l}\text { Flavonoïdes } \\
\left(\mathrm{AlCl}_{3}{ }^{\mathrm{a}}, \mathrm{NH}_{3}{ }^{\mathrm{b}}\right)\end{array}$ & $\begin{array}{c}0,04\left(\mathrm{Ja}^{\mathrm{a}}\right) ; 0,05\left(\mathrm{~B}^{\mathrm{a}}, \mathrm{Jb}^{\mathrm{b}}\right) ; 0,14\left(\mathrm{Jb}^{\mathrm{b}}\right) ; \\
0,19(\mathrm{Ja}) ; 0,25(\mathrm{Ja}) ; 0,29\left(\mathrm{Jb}^{\mathrm{b}}\right) ; \\
0,34\left(\mathrm{Ja}^{\mathrm{a}}\right) ; 0,55\left(\mathrm{~J}^{\mathrm{a}}\right) ; 0,58\left(\mathrm{Jp}^{\mathrm{b}}\right) ; \\
0,64\left(\mathrm{~J}^{\mathrm{b}}\right) ; 0,75\left(\mathrm{~J}^{\mathrm{b}}\right) ; 0,78\left(\mathrm{Ja}^{\mathrm{a}}\right) ; 0,90(\mathrm{~J}- \\
\left.\mathrm{B}^{\mathrm{a}}\right)\end{array}$ & $\begin{array}{c}0,04\left(\mathrm{~J}-O r^{\mathrm{a}}\right) ; 0,06\left(\mathrm{~B}^{\mathrm{a}}, \mathrm{Jb}^{\mathrm{b}}\right) ; 0,09(\mathrm{Ja}) ; \\
0,15\left(\mathrm{Jb}^{\mathrm{b}}\right) ; 0,19\left(\mathrm{Ja}^{\mathrm{a}}\right) ; 0,28\left(\mathrm{Jb}^{\mathrm{b}}\right) ; 0,41(\mathrm{Ja}, \\
\mathrm{b}) ; 0,56\left(\mathrm{~J}^{\mathrm{a}}\right)\end{array}$ \\
\hline & Anthocyanes $\left(\mathrm{NH}_{3}{ }^{b}\right)$ & $0,83\left(\mathrm{~B}^{\mathrm{b}}\right)$ & \\
\hline & & $\begin{array}{c}0,04(\mathrm{~J}) ; 0,08(\mathrm{~J}) ; 0,19(\mathrm{~B}) ; 0,55(\mathrm{~J}- \\
\text { Or) } ; 0,64(\mathrm{~J}) ; 0,90(\mathrm{~B})\end{array}$ & $\begin{array}{c}0,04(\mathrm{~J}-O \mathrm{Or}) ; 0,09(\mathrm{~J}) ; 0,15(\mathrm{~J}) ; \\
0,19(\mathrm{~J}) ; 0,28(\mathrm{~J}\end{array}$ \\
\hline \multirow{2}{*}{ 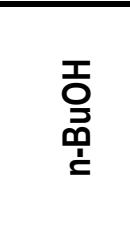 } & $\begin{array}{l}\text { Flavonoïdes } \\
\left(\mathrm{AlCl}_{3^{\mathrm{a}}}, \mathrm{NH}_{3}{ }^{\mathrm{b}}\right)\end{array}$ & $\begin{array}{c}0,05\left(J-B^{a}, J^{b}\right) ; 0,09\left(J^{b}\right) ; 0,16\left(B^{a}\right) ; \\
0,28\left(B^{a}\right)\end{array}$ & 0,09(J-Va, Jb) ; 0,19(Ba) ; 0,26(Ba) \\
\hline & $\begin{array}{c}\text { Anthocyanes }\left(\mathrm{NH}_{3}{ }^{\mathrm{b}}\right) \\
\text { Coumarines } \\
\left(\mathrm{KOH}^{\mathrm{c}}\left(\mathrm{CH}_{3} \mathrm{COO}\right)_{2} \mathrm{~Pb}^{\mathrm{d}}\right)\end{array}$ & $\begin{array}{c}0,83\left(V^{b}\right) \\
0,05\left(J-B^{d}\right) ; 0,09\left(V^{c}\right) ; 0,16\left(B^{c, d}\right) ; \\
0,28\left(B^{d}\right)\end{array}$ & $\begin{array}{c}0,14\left(B^{b}\right) ; 0,19\left(B^{b}\right) ; 0,88\left(B^{b}\right) \\
0,09\left(V^{c}, J-V^{d}\right) ; 0,19\left(B^{c, d}\right) ; 0,24\left(B^{c}\right) ; \\
0,26\left(B^{d}\right) ; 0,54\left(B^{c}\right) ; 0,84\left(B^{c}\right)\end{array}$ \\
\hline
\end{tabular}

$\mathrm{H}$ : Hexane ; $\mathrm{CHCl}_{3}$ : Chloroforme ; AcOEt : Acétate d'éthyle ; n-BuOH : n-butanol ; L-B : Liebermann-Bürchard ; $B$ : Bleue ; J: Jaune ; V: Vert ; B-V : Bleue-Bert ; Or: Orange ; V-Or: Vert-Orange Vi : Violet ; Or : Orange ; V-Vi : Vert-Violet ; J-Or : JauneOrangé ; J-Vi : Jaune-Violet ; Ter : Terpène ; St : Stérol ; Tr-Lp : Triterpène-Lupane ; B-Vi : Bleue-Violet ; B-J : Bleue-Jaune ; Vi : Violet ; B.Flu : Bleue. Fluorescente ; V-Or : Vert-Orange ; J-B : Jaune-Bleue ; B-Or : Bleue-Orange ; V-J : Vert-Jaune ; V-B : VertBleue ; J-V : Jaune-Vert.

La CCM des extraits chloroformiques a montré la coexistence des coumarines, des flavonoïdes et des anthocyanes dans les deux cultivars. Toutefois, au regard du nombre des empreintes moléculaires des flavonoïdes révélés par la solution de chlorure de d'aluminium et l'ammoniac, nous sommes fondés à croire que le cultivar LG contiendrait plus de flavonoïdes que le cultivar MK. La richesse de graines de Piper nigrum en flavonoïdes a été rapportée par certains auteurs (Grewe et al., 1970 ; Bandyopadhyay, 1990 ; Ahmad et al., 2015). La présence des alcaloïdes dans les extraits chloroformiques a été prouvée par l'usage du réactif de Dragendorff. Des travaux indiquent que le goût piquant du poivre serait dû aux alcaloïdes, notamment à la pipérine, laquelle est soupçonnée d'être responsable des vertus antimicrobienne, antiinflammatoire, hépatoprotectrice de Piper nigrum (Srinivasan, 2009 ; Ahmad et al., 2012; Tu et al., 2015). La coprésence des flavonoïdes, des anthocyanes et des coumarines a été décelée dans les extraits acétate éthyliques et n-butanoliques. En somme, il ressort de la CCM des extraits sélectifs que les graines des deux cultivars LG et MK ont présenté une diversité de métabolites secondaires d'intérêt 
pharmacologique qui justifierait l'utilisation de Piper nigrum comme condiment dans les recettes culinaires, en parfumerie et dans les préparations médicamenteuses traditionnelles (Rastogi et al., 1999; Srinivasan, 2009).

\section{Analyse quantitative}

Teneurs en phénols et flavonoïdes totaux, anthocyanes et en aglycones flavoniques

Les teneurs en phénols totaux (PT), exprimées en microgrammes équivalent d'acide gallique et celles en flavonoïdes totaux (FT) en équivalent de catéchine équivalent à 89,082 et $74,492 \mu \mathrm{gEAG} / \mathrm{g}$ et de 49,456 et $55,343 \mu \mathrm{gEC} / \mathrm{g}$ d'extrait respectivement pour MK et LG (Figure 1). Ces valeurs confirment les résultats du criblage phytochimique et témoignent de la richesse en PT et FT des graines de deux cultivars. Les teneurs en
PT $(62,3 \pm 0,08 \mu \mathrm{g} / \mathrm{g})$ sont similaires à celles mentionnées dans la littérature (Nahak \& Sahu, 2011), tandis que celles en FT sont supérieures à la teneur en FT de l'espèce indienne $(1,087 \mu \mathrm{g} / \mathrm{g}$ ) (Ahmad et al., 2015). Ce qui s'expliquerait par les conditions climatiques, les pratiques agricoles, et la maturité des graines pendant la récolte (Podsedek, 2007). Les températures élevées, l'exposition au soleil, la sécheresse et la salinité du sol stimuleraient la biosynthèse des polyphénols. Le poivrier est un hélophyte. La nouaison jusqu'à la maturité des fruits nécessite une période de sécheresse, qui semble être la justification de la forte teneur en polyphénols des graines (Falleh et al., 2008). Par ailleurs, les teneurs élevées en aglycones flavoniques démontreraient la dominance des flavonoïdes chez LG et MK (Figure 1).
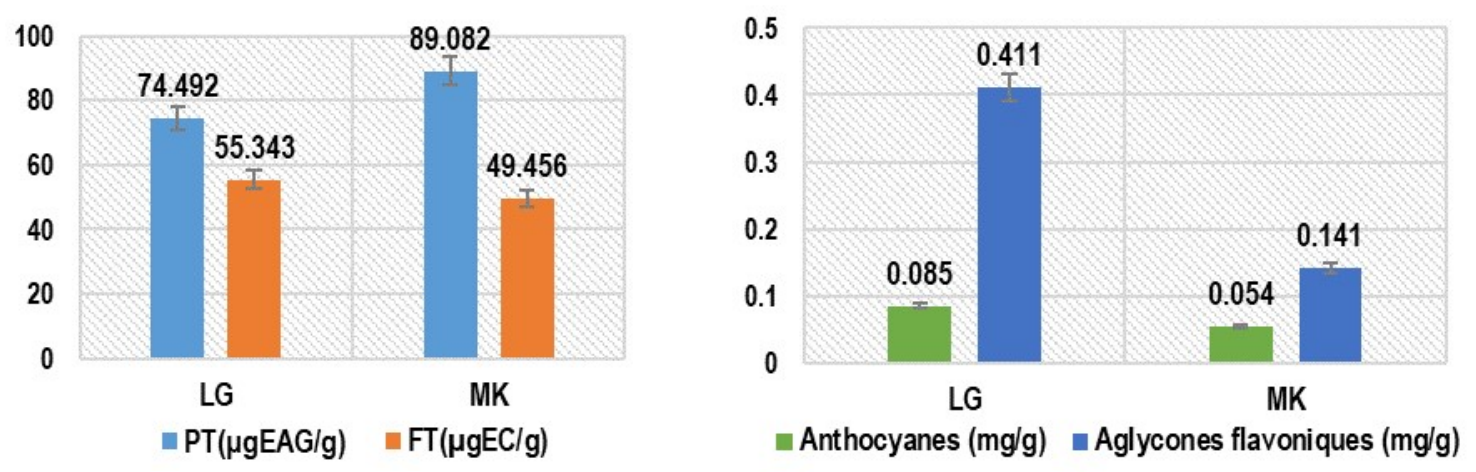

Extraits végétaux de LG et MK

Figure 1: Teneurs dans les extraits en phénols totaux (PT), flavonoïdes totaux (FT), anthocyanes et en aglycones flavoniques.

Le cultivar LG a montré les teneurs en aglycones flavoniques $(0,411 \mathrm{mg} / \mathrm{g})$ et en anthocyanes $(0,085$ $\mathrm{mg} / \mathrm{g}$ ) plus significatives que celles du cultivar MK $(0,141$ et $0,054 \mathrm{mg} / \mathrm{g})$. La présence des aglycones flavoniques chez Piper nigrum pourrait également expliquer ses propriétés hépatoprotectrice, antidiurétique, antibactérienne et antivirale (Hussein et al., 2000 ; Kumar et al., 2007).
Teneurs en tanins: Les tanins hydrolysables (TH) et condensés (TC) coexistent dans les deux cultivars, et leurs teneurs sont similaires $(7,953 \%$ (LG), 7,757\% (MK)), alors que les tanins condensés (TC) ont montré des teneurs significativement différentes 4,884\% (LG) et $6,205 \%$ (MK) (Figure 2). TH et TC sont des moyens de défense chimique que la plante utilise contre les microbes pathogènes et les herbivores. 


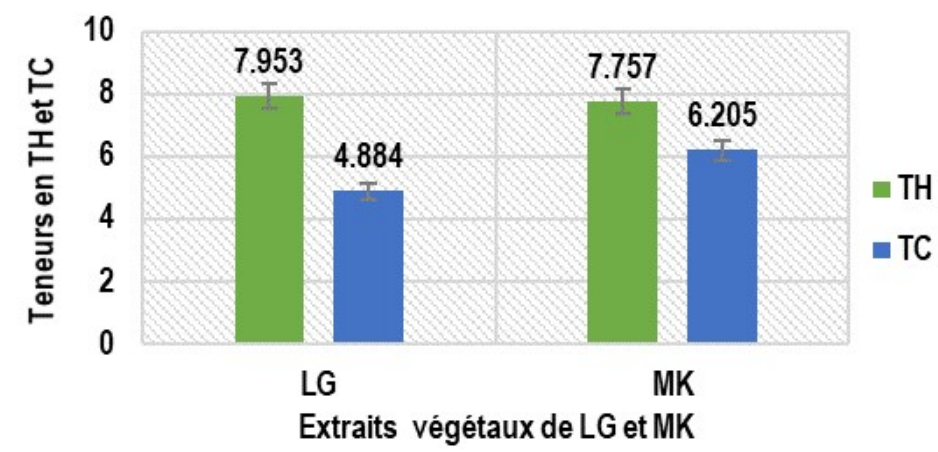

Figure 2 : Teneurs dans les extraits en tanins hydrolysables $(\mathrm{TH})$ et tanins condensés $(\mathrm{TC})$

Profil antioxydant: La figure 3 met en lumière le potentiel antioxydant de LG et MK. En effet, il ressort que LG et MK ont sensiblement réduit le DPPH concentration-dépendant. A $0,05 \mathrm{mg} / \mathrm{ml}, \mathrm{LG}$ et MK réduisent de façon modérée le $\mathrm{DPPH}$ avec des pourcentages de réduction de $64,983 \%$ et $55,022 \%$, respectivement, et ce, comparativement à la quercétine $(85,714 \%)$. Toutefois, à cette concentration, le potentiel antioxydant de LG serait plus significatif que celui de MK vis-à-vis du DPPH ; ce qui serait en accord avec leurs compositions phytochimiques préliminaires respectives établies (Tableau 1).

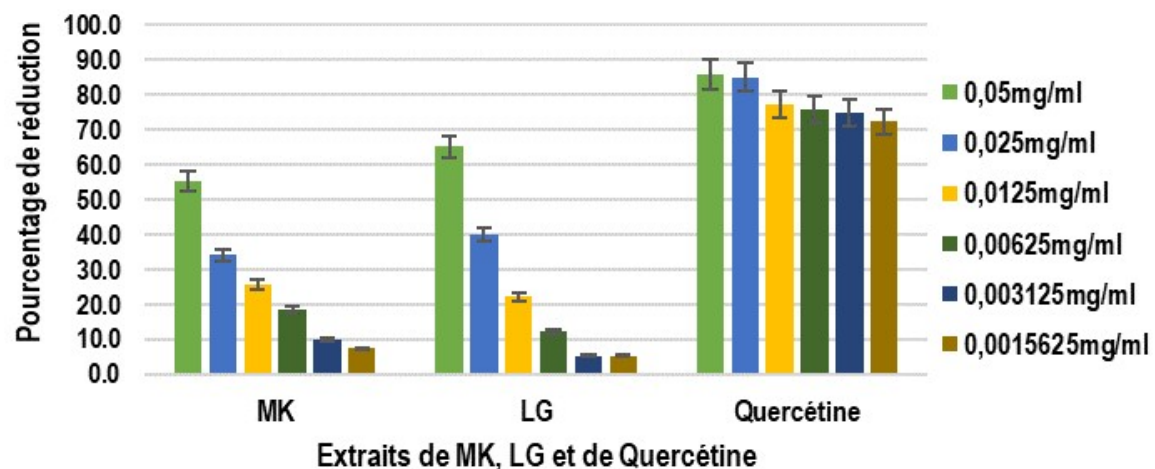

Figure 3 : Activité antioxydante des poivriers et de la Quercétine

La réduction du DPPH rend compte de la présence de phytocomposés bioactifs réducteurs par don d'hydrogène, au nombre desquels se trouvent les phytophénols notamment. II semble clair qu'il existe une corrélation entre la teneur en PT et l'activité antiradicalaire. Ce constat a été fait par Andriana et al., (2019) dans une étude sur Piper nigrum. Les alcaloïdes manifestent une activité antioxydante. Tu et al., (2015) ont démontré que les alcaloïdes en particulier la

\section{CONCLUSION}

Le présent travail a montré que les deux cultivars de Piper nigrum, domestiqués en Côte d'Ivoire contiennent des phytocomposés antioxydants. La coprésence des alcaloïdes, des stérols, des terpènes, des coumarines, des tanins et des flavonoïdes a été mise en évidence à l'aide de la CCM. L'étude quantitative des phytocomposés a révélé la présence significative de pipérine, la pipérettine et la pipérettyline, seraient responsables du pouvoir antioxydant de Piper nigrum.. La détermination de la $\mathrm{CE}_{50}$ a permis de mieux apprécier le potentiel anti radicalaire des échantillons testés. En effet, plus cette valeur est petite, et plus est meilleure l'activité anti radicalaire (Falleh et al., 2008). Les valeurs de la $C E_{50}$ de LG $(1,209)$, MK $(1,432)$ sont inférieures à celles de la quercétine $(0,05)$.

polyphénols, flavonoïdes, anthocyanes, aglycones flavoniques, tanins hydrolysables et condensés. L'évaluation du potentiel oxydant par rapport au DPPH a montré $L G$ et $M K$ manifesteraient une activité antioxydant, et ce, en rapport de cause à effet avec leurs compositions phytochimiques. Par ailleurs, il atteste qu'au regard de la composition phytochimique 
et du potentiel antioxydant des cultivars de Piper nigrum qu'un début vers la conception d'un

\section{RÉFÉRENCES}

Achinewu SC, Aniena MI, Obomanu FG, 1995. Studies on spices of food value in the south states of Nigeria: antioxidant properties. Journal of African Medicinal Plants 18:135-139.

Ahmad N, Fazal H, Ayaz M, Abbasi BH, Mohammad I, Fazal L, 2011. Dengue fever treatment with Carica papaya leaves extracts. Asian Pacific Journal of Tropical Biomedicine 1 (4): 330-333.

Ahmad N, Fazal H, Abbasi BH, Farooq S, Ali M, Khan MA, 2012. Biological role of Piper nigrum $L$. (Black pepper): A review. Asian Pacific Journal of Tropical Biomedicine 2 (3): 1945-1953.

Ahmad A, Husain A, Mujeeb M, Khan SA, Alhadrami HAA, Bhandari A, 2015. Quantification of total phenol, flavonoid content and pharmacognostical evaluation including HPTLC fingerprinting for the standardization of Piper nigrum L. fruits. Asian Pacific Journal of Tropical Biomedicine 5(2): 101-107.

Andriana Y, Xuan TD, Ngoc Quy T, Hoang-Dung T, Quang-Tri L, 2019. Biological Activities and Chemical Constituents of Essential Oils from Piper cubeba Bojer and Piper nigrum L. Molecules 24 (1876): 1-16.

Ao P, Hu S, Zhao A, 1998. Essential oil analysis and trace element study of the roots of Piper nigrum L. Zhongguo Zhong Yao Za Zhi 23: 4263.

Bandyopadhayay C, Narayan VS, Variyar PS, 1990. Phenolics of green pepper berries (Piper nigrum L.) Journal of Agricultural and Food Chemistry 38, 1696-1699.

Békro YA, Mamyrbekova Békro JA, Boua BB, Tra Bi $\mathrm{FH}$, Ehilé $\mathrm{EE}, 2007$. Etude ethnobotanique et screening phytochimique de Caesalpinia benthamiana (Baill.) Herend. Et Zarucchi (Caesalpiniaceae). Sciences et Nature 4 (2): 217-225.

Borget M, 1991. Les plantes tropicales à épices, 1er édition, Maisonneuve \& Larose Paris. $182 p$.

Chen W, Dou H, Ge C, Congfa L, 2011. Comparison of volatile compounds in Pepper (Piper nigrum L.) by simultaneous distillation extraction (SDE) and GC-MS. Advanced Material Research 236: 2643-2646.

Dif MM, Benchiha $\mathrm{H}$, Mehdadi Z, Benali-Toumi $\mathrm{F}$, Benyahia M, Bouterfas K, 2015. Étude complément alicamentaire pour l'humeur et la santé a été initié.

quantitative des polyphénols dans les différents organes de l'espèce Papaver rhoeas L. Phytothérapie 13 (5): 324-319.

Dohou N, Yamni K, Tahrouch S, Idrissi Hassani LM, Badoc A, Gmira N, 2003. Screening Phytochimique d'une endémique ibéromarocaine Thymelaea lithroïdes. Bulletin de la Société de Pharmacie Bordeaux 142: 61-78.

Falleh $H$, Ksouri R, Abdelly C, 2006. Activité antioxydante et contenu en polyphénols dans les différents organes de l'artichaut sauvage Cynara cardunculus. Revue des régions arides Tunisie. $344 p$

Espín JC, Soler-Rivas C, Wichers HJ, 2000. Characterization of the total free radical scavenger capacity of vegetable oils and oil fractions using 2,2-diphenyl-1-picrylhydrazyl radical. Journal of Agricultural and Food Chemistry 48(3): 648-56

Ganesh P, Suresh Kumar R, Saranraj P, 2014. Phytochemical analysis and antibacterial activity of Pepper (Piper nigrum L.) against some human pathogens. Central European Journal of Experimental Biology 3(2): 36-41.

Grewe R, Freist W, Newmann J, Kersten S, 1970. Uber die inhaltsstoffe des schwarzen pfeffers. Chemische Berichte 103: 3572-3580.

Heilerová L, Bućkova M, Tarapćik P, Silhár S, Labuda J, 2003. Comparison of antioxydative activity data for aqueous extracts of Lemon balm (Melissa officinalis L.), Oregano (Origanum vulgare L.), Thyme (Thymus vulgaris L.), and Agrimony (Agrimonia eupatoria L.) obtained by conventional methods and the DNA-based biosensor. Czech Journal Food Science 21(2): 78-84.

Hussein G, Miyashiro $H$, Nakamura N, Hattori M, Kakiuchi N, Shimotohno K, 2000. Inhibitory effects of Sudanese medicinal plant extracts on hepatitis $\mathrm{C}$ virus (HCV) protease. Phytotherapy research $14:$ 510-516.

Kabran G, 2007. Screening phytochimique et détermination de flavonoïdes totaux des extraits des feuilles de Secamone afzelii (Asclepiadaceae). Mémoire de maîtrise des Sciences Fondamentales et Appliquées; 
Université d'Abobo Adjamé (Côte d'Ivoire). 33 p.

Kumar S, Singhal V, Roshan R, Sharma A, Rembhotkar GW, Ghosh B, 2007. Piperine inhibits TNF-a induced adhesion of neutrophils to endothelial monolayer through suppression of NF-K and IKB kinase activation. European Journal of Pharmacology 575: 177-186.

Ladigina EY, Safronich LN, Otriacheva VE, Balandina $I A$, Grinkevich NI, Sorokina AA, Glizin VI, Molodojnikova LM, Mitin YS, Samilina IA, Ermakova VA, 1983. Analyse chimique des plantes médicinales, Edition Moskva vischaya chkola, $172 \mathrm{p}$.

Lebreton P. et Jay M, 1967. Sur l'analyse qualitative et quantitative des flavonoïdes. Chemical Analysis Paris 49 (7): 375-383.

Liu H, Zheng J, Liu P, Zeng F, 2018. Pulverizing processes affect the chemical quality and thermal property of black, white, and green pepper (Piper nigrum L.). Journal of Food Science and Technology 55 : 2130-2142.

N'gaman KCC, 2013. Étude phytochimique et effet d'extraits de Gmelina arborea Roxb. (Verbenaceae) de Côte d'Ivoire sur la stabilité osmotique d'érythrocytes. Thèse de doctorat, Université Nangui Abrogoua, Abidjan, 152 p.

N'Guessan AHO, Dago DCE, Mamyrbékova-Békro JA, Békro YA, 2011. Teneurs en composés phénoliques de 10 plantes médicinales employées dans la tradithérapie de l'hypertension artérielle, une pathologie émergente en Côte d'Ivoire. Revue de génie industriel 6: 55-61.

Nair RR. and Gupta SD, 2003. Somatic embryogenesis and plant regeneration in black pepper (Piper nigrum L.). I. Direct somatic embryogenesis from tissues of germinating seeds and ontogeny of somatic embryos. Journal of horticultural science \& biotechnology 78 (3): 416-421.

Nahak G. and Sahu RK, 2011. Phytochemical Evaluation and Antioxidant activity of Piper cubeba and Piper nigrum. Journal of Applied Pharmaceutical Science 1(08): 153-157.

Podsędek A, 2007. Natural antioxidants and antioxidant capacity of brassica vegetables: A review. LWT-Food Science and Technology 40(1): 111.

Rastogi RP. and Mehrotra BN, 1998. Compendium of Indian Medicinal Plants. 2nd Reprint, Central
Drug Research Institute, Lucknow and National Institute of Science Communication, Council of Scientific and Industrial Research, New Delhi, 434-436.

Salehi B, Zakaria ZA, Gyawali R, Salam Al, Rajkovic J, Shinwari ZK, Khan T, Sharifi-Rad J, Ozleyen A, Turkdonmez E, Valussi M, Tumer TB, Fidalgo LM, Martorell M, Setzer WN, 2019. Piper Species: A Comprehensive Review on Their Phytochemistry, Biological Activities and Applications. Review Molecules 24 (1364) : 1118.

Scott IM, Jensen HR, Philogene BJR, Arnason JT, 2008. A review of Piper spp. (Piperaceae) Phytochemistry, insecticidal activity and mode of action. Phytochemistry Review 7: 65-75.

Singleton VL, Orthofer R, Lamuela-Raventos RM, 1999. Analysis of total phenols and other oxidation substrates and antioxidants by means of FolinCiocalteu reagent. Methods Enzymology 299, 152-178.

Sladjana MS, Gordana SĆ, Jasna MČB, Sonja MD, 2012. Comportement cinétique de l'activité de balayage des radicaux DPPH' d'extraits de déchets de tomate. Journal of the Serbian Chemical Society 77 (10): 1381-1389.

Srinivasan K, 2009. Black Pepper (Piper nigrum) and Its Bioactive Compound, Piperine; Molecular Targets and Therapeutic Uses of Spices: 1-40.

Tu Y, Zhong Y, Du H, Luo W, Wen Y, Li Q, 2015. Anticholinesterases and antioxidant alkamides from Piper nigrum fruits. Natural Product Research 30(17): 1945-1949.

Xiang CP, Han JX, Li XC, Li YH, Zhang Y, Chen L, Qu Y, Hao CY, Li HZ, Yang CR, 2017. Chemical composition and acetylcholinesterase inhibitory activity of essential oils from Piper species. Journal of Agricultural and Food Chemistry $65:$ 3702-3710. 